Mots. Les langages du politique

96 | 2011

Les discours politiques. Approches interactionnistes et multimodales

\title{
Les discours politiques. Approches interactionnistes et multimodales
}

Hugues Constantin de Chanay et Olivier Turbide

\section{(2) OpenEdition \\ Journals}

Édition électronique

URL : https://journals.openedition.org/mots/20170

DOI : $10.4000 /$ mots. 20170

ISSN : 1960-6001

Éditeur

ENS Éditions

Édition imprimée

Date de publication : 5 septembre 2011

Pagination : 5-12

ISBN : 978-2-84788-316-9

ISSN : 0243-6450

Référence électronique

Hugues Constantin de Chanay et Olivier Turbide, « Les discours politiques. Approches

interactionnistes et multimodales ", Mots. Les langages du politique [En ligne], 96 | 2011, mis en ligne le 05 septembre 2013, consulté le 22 avril 2022. URL : http://journals.openedition.org/mots/20170 ;

DOI : https://doi.org/10.4000/mots.20170 


\section{Les discours politiques. Approches interactionnistes et multimodales}

Envisager les discours politiques dans une perspective interactionniste, c'est considérer que la nature politique d'un discours s'établit en fonction de la relation instaurée entre les acteurs et auteurs de ces discours et les différentes catégories de récepteurs. Comme le rappelle Charaudeau (2005, p. 30), ce n'est pas le locuteur qui fait qu'un discours est politique, ni le contenu de son discours, ni les lieux de son expression ou les types d'activités, mais c'est la situation d'interaction. Dans cette perspective, partagée par van Dijk (1997, p. 15), est politique tout discours qui s'inscrit dans un type d'activité de communication (conférence de presse, débat, allocution partisane, etc.) dont la conjonction du cadre spatio-temporel, des statuts et des rôles des acteurs, des finalités et des actions produit des effets politiques possibles.

Sur le plan analytique, cette conception interactionniste permet d'élargir le champ d'étude à des activités médiatiques non traditionnelles comme les émissions d'info-divertisssement (Eriksson, 2009; Hamo, 2010; Turbide, à paraître) dont l'effet politique d'identification du public est reconnu (Horton, Wohl, 1986), mais aussi à des catégories d'acteurs non médiatisés qui, sans être des professionnels politiques, visent à travers leurs actions à s'approprier la légitimité de parler, d'agir au nom des citoyens et de les mobiliser autour d'un projet. Pensons aux élus municipaux, aux représentants étudiants ou aux chefs syndicaux.

Sur le plan épistémologique, cette conception interactionniste des discours politiques suggère que le sens d'une prestation n'est pas entièrement pris en charge par les acteurs et auteurs des discours et diffusé à des récepteurs qui seraient passifs, mais se construit dans ce rapport entre représentants politiques et récepteurs (voir Goffman, 1981, p.137-138). Même lorsque ces derniers n'interviennent pas directement dans le développement des échanges comme lors d'interactions médiatiques ou de prises de parole publiques, ils agissent comme des «tiers symbolisants» (Quéré, 1982) capables de nourrir les attentes quant au déroulement de l'activité (Relieu, Brock, 1995, p. 101). La 
coconstruction interactionnelle s'établit à travers les images que l'un se fait de l'autre. Ainsi, en fonction de la représentation que l'acteur politique se fait des gens à qui il s'adresse, des réactions qu'il anticipe s'il dit telle chose ou telle autre, des valeurs et des croyances qu'il leur prête, il ajustera ses comportements, l'intonation de sa voix, le choix des mots, l'expression de son visage, etc. Dans ce cadre, le rôle du représentant politique est de construire une situation offrant au public la possibilité d'intervenir «virtuellement» dans l'échange mis en scène en les interpellant, en les ratifiant, en les impliquant discursivement, etc. (Tolson, 2006, p.10)

Plusieurs études (Fairclough, 1998; Clayman, 2004; Martel, 2010) montrent d'ailleurs comment ce mode interactionniste de livraison d'un discours affecte les prestations des représentants politiques, révélant différentes stratégies pour faire du public un participant ratifié ${ }^{1}$ de l'échange. D'une part, les interpellations visuelles (axe de caméra les yeux dans les yeux) et discursives (adresses directes au public), de même que les recours à des formes pronominales inclusives pour marquer l'identification du locuteur au public, l'adoption d'un registre de discours moins formel, sont quelques-uns des moyens communicationnels utilisés pour reproduire les conditions d'une interaction en face à face (Isotalus, 1998; Scollon, 1998). D'autre part, l'intégration de mises en scène interactionnelles pour présenter l'information politique (entrevues, discussions, panels, débats, conférences de presse), au détriment du format de livraison traditionnelle (impersonnel, monologal, statique), renvoie à un cadre interactionnel où le discours résulte d'ajustements permanents et de négociations continues entre les participants (Hutchby, 2006, p.12-13). Cette double évolution des pratiques communicationnelles nécessite un ajustement des outils théoriques et analytiques pour rendre compte des formats interactionnels à travers lesquels l'information politique est souvent présentée.

Une littérature abondante provenant des champs de l'analyse conversationnelle, de l'analyse du discours, de la sociologie et de la linguistique ont permis d'établir les fondements théoriques d'une analyse interactionniste des discours politiques. Pour des raisons de facilité d'accès aux données audiovisuelles, les interactions médiatiques ont été largement privilégiées. L'analyse des discours politiques s'intègre au champ du broadcast talk (voir Scannell, 1991; Hutchby, 2006; Tolson, 2006). Dans la lignée des travaux de Goffman (1973 [1959]) sur l'interaction et de l'ethnométhodologie de Garfinkel (1967), Heritage, Clayman et Zimmerman, dans un article paru en 1988 , sont parmi les premiers à analyser les prises de parole médiatiques sous l'angle de leur organisation séquentielle, révélant que, tout comme en conversation, l'alternance des tours de parole structure

1. Pour Goffman (1981, p.9-10), "those ratified participants who are adressed, that is, oriented to by the speaker in a manner to suggest that his words are particularly for them, and that some answer is therefore anticipated from them $»$. 
avec force la construction des messages. Étudiant les interviews médiatiques de représentants politiques, ils montrent comment le sens s'élabore tour après tour de manière contingente et locale. Chaque prise de parole est l'objet d'une négociation permanente et d'ajustements réciproques. Cette logique de coconstruction n'est pas spécifique à ce type d'activité. Que ce soit sur la scène médiatique ou sur la scène publique, les représentants politiques doivent adopter des comportements culturellement et socialement adéquats et efficaces, endosser certains rôles établis en fonction de ce que la situation exige, composer avec l'image que revendique autrui tout en tentant d'imposer la sienne. En somme, leur capacité à montrer qu'ils savent se comporter discursivement (répondre aux attaques, défendre leurs propositions) et qu'ils sont dignes de confiance est le fruit d'une dynamique complexe d'adaptations in situ.

L'étude de cette construction locale du sens a été longtemps réduite à ses seuls aspects linguistiques. Bien souvent dans les analyses de discours, la posture adoptée a consisté à identifier la dimension verbale des comportements comme un système autonome des autres modes sémiotiques, comme le principal lieu d'émergence des significations et comme le poste d'observation privilégié pour appréhender des phénomènes variés de communication politique. Le postulat sous-jacent est que les comportements verbaux constituent le support dominant du discours alors que la voix, le regard, les gestes et les mimiques seraient des indices transversaux pour la compréhension. Dans cette logique, la prise en compte exhaustive des dimensions non verbale et paraverbale ${ }^{2}$ ne ferait pas émerger une nouvelle interprétation des échanges, mais viendrait simplement nuancer, compléter, enrichir les informations accessibles sur le plan verbal.

Or, les travaux en analyse conversationnelle d'orientation ethnométhodologique ont montré comment l'organisation de l'alternance des tours de parole procède d'une mécanique fine et complexe qui engage l'ensemble des ressources sémiotiques de l'agir. L'identification par un locuteur d'une place pertinente pour prendre la parole est établie en fonction de l'interprétation qu'il fait du tour précédent (Sacks, Schegloff, Jefferson, 1974), une interprétation qui est nourrie largement des manifestations voco-mimo-posturo-gestuelles de l'interlocuteur. Pour l'analyste, l'observation de ces manifestations permet d'avoir une prise sur la façon dont les interactants comprennent et interprètent les contributions de leurs interlocuteurs et ajustent leurs comportements en conséquence (Trognon, 1990, p. 220).

Le cas devenu classique du débat politique américain entre Kennedy et Nixon en 1960, où les récepteurs qui avaient écouté les échanges à la radio ont donné gagnant Nixon, alors que ceux qui les avaient regardés à la télévision,

2. La terminologie généralement employée pour désigner ces modes sémiotiques, «paraverbal» pour les données prosodiques et «non verbal » pour les aspects kinésiques de la communication, témoigne d'une conception verbocentriste de ces ressources de l'agir, assujeties au verbal dans une relation de dépendance. 
percevant l'attitude corporelle déficiente de Nixon, ont donné largement Kennedy gagnant, témoigne de l'apport des comportements paraverbaux et non verbaux à la construction du sens.

Le corps n'est pas un simple diffuseur du Verbe, lequel s'y ferait chair à la faveur de la présence physique de ses émetteurs [...]. Au contraire, la présence des débatteurs en chair et en os [...] délivre un apport communicatif propre, non seulement parce que les corps constituent les supports d'inscription non neutres sur lesquels le verbal, d'une certaine manière, s'écrit et s'actualise (c'est l'actio de la rhétorique classique), mais encore parce qu'ils écrivent eux-mêmes au sein de la partition communicative globale leurs propres lignes, non moins essentielles et différenciées que ne le seraient celles d'un ou même de plusieurs instruments de musique appelés à figurer dans un effectif orchestral. (Constantin De Chanay, 2009, p. 62)

Dans cette perspective holistique, l'analyse des interactions n'est pas réductible à la prise en compte du verbal, ni à l'étude séparée de différents modes sémiotiques. Parce que l'ensemble des ressources de l'agir fonctionne en synergie, le sens prend forme de la combinaison et de l'agrégation des mots prononcés, du rythme et du ton de la voix, des mimiques faciales et des gestes accomplis dans un contexte donné. II ne s'agit pas d'analyser les différents systèmes sémiotiques comme des entités autonomes, ayant leur propre mode de fonctionnement et système de significations, mais de comprendre l'interrelation de ces ressources. On passe alors de la «multicanalité de la communication » à la « multimodalité de l'action» dont les travaux de Kraft et Dausendschön-Gay (2001), de Goodwin (2000) et de Kress et van Leeuwen (2001) ont contribué à établir les fondements analytiques et méthodologiques (voir Filliettaz, 2009, p. 51).

Dans le domaine de l'analyse des discours politiques, même si on a coutume de soutenir que l'action politique se réalise pour une large part à travers la parole, les travaux récents de Bull (2003), de Kerbrat-Orechhioni (2010) et de Constantin de Chanay (2009), pour ne nommer que ceux-là, permettent de voir comment la prise en compte de la multimodalité des discours politiques s'affiche comme une nécessité. Que ce soit sur la scène médiatique ou sur la scène publique, c'est le corps des représentants politiques plus encore que les mots qui est mis en scène et spectacularisé. D'une part, la combinaison des marques voco-mimo-posturo-gestuelles personnalise l'acteur, construit son unicité, facilitant l'adhésion du public à son personnage, public qui le reconnaît immédiatement par sa voix, son attitude corporelle, ses postures, etc. D’autre part, la mise en voix et en gestes du discours constitue le lieu privilégié de la construction de l'ethos du représentant politique. À chaque fois que l'acteur communique, voire à chaque fois qu'il est en présence d'autrui, se forme chez ses interlocuteurs une certaine image de lui-même.

La recherche en analyse des discours politiques s'inscrivant dans cette double dimension interactionniste et multimodale n'est qu'à ses débuts. Si les méthodes de transcription des différents modes sémiotiques et leur annota- 
tion se sont raffinées au fil des ans (voir Mondada, 2007), leur alignement - le déploiement temporel des comportements para- et non verbaux, en synchronie, avec la parole - et surtout l'intégration de l'ensemble des observables dans une interprétation unifiée du discours pose toujours un défi. Au surplus, les mimiques, les comportements gestuels, les postures, la voix, parce que moins bien balisés sémantiquement - leur décodage est généralement plus imprécis que les comportements verbaux -, doivent être interprétés avec précaution et toujours en lien avec le flux verbal (Kerbat-Orecchioni, 2010, p. 43-44). Les trois études présentées dans ce dossier participent à cet effort de recherche visant à articuler ces deux dimensions du discours politique dans le but d'enrichir notre compréhension de ces microphénomènes communicationnels. Elles mettent en lumière l'apport des comportements voco-posturo-mimo-gestuels à l'interprétation des discours politiques, qu'ils soient médiatiques (débat télévisé, talk-show) ou publics (débat public).

L'étude de Marion Sandré sur les rires et les sourires dans un débat télévisé rappelle comment ces mimiques sont motivées par le discours d'autrui, soit en y réagissant, soit en l'anticipant. Même s'ils sont construits localement en situation, Sandré montre que lorsqu'il s'agit de professionnels de la politique comme les candidats à l'élection présidentielle de 2007 en France, l'usage de ces comportements coverbaux est avant tout stratégique, savamment travaillé pour produire l'image que le candidat veut laisser de sa personne et de son adversaire. Loin de n'être qu'une composante phatique de la parole, qu'un accompagnateur, les rires/sourires interviennent dans l'interprétation des discours, produisant des effets de valorisation de soi et de dévalorisation d'autrui tout en participant à la construction du lien entre le représentant politique et les téléspectateurs. De manière spécifique, Sandré présente deux catégories de rires/sourires : 1) ceux réactifs, motivés par le discours de l'interlocuteur; 2) ceux initiés par le locuteur. Pour la première catégorie, l'auteur distingue l'effet consensuel des rires/ sourires de l'effet dissensuel. Alors que le premier type renvoie à la contribution de ces mimiques à l'établissement d'un rapport de connivence avec l'interlocuteur, allant du rire de complicité au sourire d'accord contraint, le second type d'effet concerne les rires quasi linguistiques qui, par leur seule production, signalent le désaccord du locuteur. Pour la seconde catégorie, à travers la présentation des effets atténuateur et amplificateur, l'auteur illustre comment ces rires/sourires permettent au locuteur de modaliser les reproches adressés selon l'image qu'il veut projeter : soit attaquer tout en se montrant courtois, affaiblissant ainsi la portée de l'agression, soit, à l'inverse, prendre clairement le parti de défaire l'autre, de le discréditer. Sur ce dernier aspect, les exemples présentés témoignent bien de la force agressive des sourires qui semble se substituer à la parole. Le dernier effet de cette catégorie, l'effet révélateur, renvoie à un sentiment particulier (d'autosatisfaction, par exemple), qui émerge de la mise en relation du flux verbal avec des rires/sourires sans être explicitement verbalisé. 
De façon similaire, Ricardo Peñafiel traite de la question de la construction de l'ethos en interaction, mais cette fois dans un talk-show vénézuélien animé par le président du pays, Hugo Chávez. L'auteur cherche à montrer comment l'efficacité et la crédibilité de l'ethos populaire revendiqué par Chávez résultent de la convergence d'éléments de mise en scène spécifiques à l'émission Aló Presidente et d'indices interactionnels, verbaux, vocaux et gestuels performés devant public. Cet ethos qui favorise l'identification du peuple se fonde autant sur une proximité physique (Chávez va à la rencontre des gens, sympathise avec eux, s'habille comme eux, les touche de la main) que sur une proximité symbolique dont le recours à des formes pronominales inclusives (" nous » collectif), l'adoption d'un registre de discours moins formel, d'un ton de confidence et d'une position énonciative se confondant avec celle du peuple constituent les principales marques. Sur le plan interactionnel, les signes d'assentiment et les sourires réciproques contribuent à l'établissement d'un rapport de connivence avec les interlocuteurs à travers lequel les téléspectateurs peuvent se reconnaître. En outre, l'auteur rappelle que l'efficacité de cette construction multimodale tient aussi à la capacité de Chávez à faire cohabiter, au sein de son discours, cet ethos populaire avec l'ethos présidentiel de celui qui répond aux demandes de ce peuple. Une construction qui n'est pas le fruit d'un travail spontané, mais s'appuie et confirme un prédiscours qui articule déjà cette double composante éthique.

S'inscrivant résolument dans une perspective d'analyse conversationnelle, le travail de Jérôme Jacquin témoigne de la richesse et de la complexité d'une analyse détaillée de la construction du sens en situation qui fait appel autant aux ressources linguistiques et interactionnelles que prosodiques et mimogestuelles. Décrivant comment les participants s'orientent vers la réalisation d'actions conjointes en vue de « débattre », l'auteur articule des cadres conceptuels variés afin d’illustrer la congruence et la complémentarité des différents modes sémiotiques convoqués. La représentation de sens commun de l'activité de débattre comme quelque chose de désorganisé où tout peut se produire (voir Clayman, 2008, p. 85) est déconstruite par l'étude fine des marques de complétude des tours de parole. Celles-ci fournissent aux participants les matériaux pour déceler la fin de la Question du débat, et pour s'orienter vers l'enchaînement interactionnel d'une Proposition et d'une Opposition, selon le modèle de la «situation argumentative » défini par Plantin (2005). La description de cette organisation séquentielle est proposée pour un extrait d'un débat public relativement «artisanal » parce que non médiatisé et n’impliquant pas des professionnels de la politique, ce qui confère au corpus son originalité. Cependant, on peut penser que le patron interactionnel dégagé serait similaire pour d'autres formes de débats publics et médiatiques, donnant un potentiel de généralisation à l'analyse multimodale de Jacquin.

En bref, les trois analyses rassemblées dans ce dossier explorent différentes façons d'articuler les dimensions interactionnelle et multimodale des discours 
politiques. Dès lors que l'on passe d'une perspective transmissionniste de la communication à une perspective relationnelle et actionnelle (voir Filliettaz, 2002), il importe d'examiner tout microphénomène qui modifie et qui conditionne l'interprétation des discours politiques. Si, dans un premier temps, une large part des études s'est concentrée sur l'analyse des interactions verbales, l'élargissement aux autres modes sémiotiques permet de mieux comprendre ce qui fonde le pouvoir d'identification, de persuasion et de mobilisation de certains représentants politiques.

\section{Références}

Bull Peter, 2003, The Microanalysis of Political Communication. Claptrap and Ambiguity, Londres, Psychology Press.

Charaudeau Patrick, 2005, Le discours politique. Les masques du pouvoir, Paris, Vuibert.

CLAYMAN Steven, 2004, "Arenas of interaction in the mediated public sphere », Poetics, no 32 , p. 29-49.

- 2008, «Talk in interaction as a locus for media studies», L'analyse linguistique des discours médiatiques. Entre sciences du langage et sciences de la communication, M. Burger éd., Québec, Nota Bene, p. 83-109.

CONSTANTIN DE ChAnAY Hugues, 2009, "Corps à corps en 2007. Nicolas Sarkozy face à Ségolène Royal », Itinéraires. Littérature, textes et cultures, nº 1, p. 61-80.

ERIKSSON Göran, 2009, "The management of applause and laughter in live political interviews», Media, Culture \& Society, vol. XXXI, nº 6, p. 901-920.

FAIRCLOU GH Norman, 1998, Critical discourse analysis. The critical study of language, Londres, New York, Longman.

FILLIETTAZ Laurent, 2002, La parole en action. Éléments de pragmatique psycho-sociale, Québec, Nota Bene.

- 2009, "Les représentations du travail dans des réunions de relève de poste en milieu industriel. Une analyse multimodale», Langage, objets enseignés et travail enseignant en didactique du français, S. Canelas-Trevisi éd., Grenoble, Ellug, p. 47-70.

GARFIn Kel Harold, 1967, Studies in Ethnomethodology, Cambridge, Polity Press.

GoFFMAN Erving, 1973 [1959], La mise en scène de la vie quotidienne. La présentation de soi, Paris, Minuit.

- 1981, Forms of Talk, Philadelphie, Philadelphia University Press.

Goodwin Charles, 2000, "Action and embodiment within situated human interaction », Journal of Pragmatics, n³ 32, p. 1489-1522.

Hamo Michal, 2010, "Surviving the "mock interview". Challenges to political communicative competence in comtemporary discourse», Media, Culture \& Society, vol.XXXII, n² 2, p. 247-266.

Heritage John, Clayman Steven, Zimmerman Donald, 1988, "Discourse and message analysis. The micro-structure of mass media messages», Advancing Communication Science : Merging Mass and Interpersonal Processes, R. P. Hawkins, 
J. M. Wiemann, S. Pingree éd., Sage Annual Reviews of Communication Research, vol.XVI, Londres, Sage, p. 77-109.

HoRTON Donald, Wohl Richard, 1986, « Mass communication and para-social interaction. Observation on intimacy at a distance », Inter/Media. Interpersonal Communication in a Media World, R. Catchcart, G. Gumpert éd., New York, Oxford University Press, p. 185-206.

Hutchby Ian, 2006, Media Talk. Conversation Analysis and the Study of Broadcasting, Maidenhead, Open University Press.

Isotalus Pekka, 1998, "Television performance as interaction», Nordicom Review, vol.XIX, nº1, p.175-183.

Kerbrat-OrecchionI Catherine, 2010, "Pour une analyse multimodale des interactions orales. L'expression des émotions dans les débats politiques télévisuels», Cadernos de Letras da UFF, $\mathrm{n}^{\circ}$ 40, p.17-45.

KRAFT Ulrich, DAUSENDSCHÖN-GAY Ulrich, 2001, «La multidimensionnalité de l'interaction. Textes, gestes, et le sens des actions sociales», Marges linguistiques, n² 2, p. 120-139.

KRESS Gunther et VAN LeEUVwen Theo, 2001, Multimodal Discourse. The Modes and Media of Contemporary Communication, Londres, Arnold.

MARTEL Guylaine, 2010, "La performance communicationnelle en contexte médiatique. L'exemple du débat politique télévisé», Mots. Les langages du politique, $\mathrm{n}^{\circ}$ 92, Rumeurs en politique, p. 83-101.

Mondada Lorenza, 2007, «Multimodal resources for turn-taking. Pointing and the emergence of possible next speakers », Discourse Studies, n 9, vol. II, p. 194-225.

Plantin Christian, 2005, L'argumentation, Paris, PUF.

QUÉRÉ Louis, 1982, Des miroirs équivoques. Aux origines de la communication moderne, Paris, Aubier-Montaigne.

Relieu Marc, BRock Franck, 1995, "L'infrastructure conversationnelle de la parole publique», Politix, nº31, p. 77-112.

SACKS Harvey, SCHEgloff Emanuel, Jefferson Gael, 1974, «A simplest systematics for the organisation of turn-taking for conversation », Language, n 50 , p. 696-735.

SCANNELL Paddy, 1991, «Introduction : the relevance of talk», Broadcast Talk, P. Scannell éd., Londres, Sage, p. 1-13.

Scollon Ron, 1998, Mediated Discourse as Social Interaction. A Study of News Discourse, Londres, Longman.

Tolson Andrew, 2006, Media Talk. Spoken Discourse on TV and Radio, Édimbourg, Edinburgh University Press.

TROGNON Alain, 1990, "La fixation de l'interprétation des énoncés dans l'interaction conversationnelle», Les sciences cognitives en débat, G. Vergnaud éd., Paris, CNRS, p. 207-218.

TURBIDE Olivier, à paraître, «Discours politique et gestion de la confrontation lors d'un talkshow et d'une émission d'affaires publiques», La parole politique en confrontation dans les médias, M. Burger, J. Jacquin, R. Micheli éd., Bruxelles, De Boeck Université.

VAN DIJK Teun, 1997, «What is political discourse analysis? », Belgian Journal of Linguistics, $\mathrm{n}^{0} 11, \mathrm{p} .11-52$. 